in copying, the wrong data were included in the table referred to, and it is the purpose of this note to present the correct ones. The following table should be substituted for that given in the article:

Table IV.-Specific Conductance (L) $\times 10^{\circ}$ of Mixtures of Pyro- and OrthoPHOSPHORIC ACIDS AT $18^{\circ}$.

\begin{tabular}{|c|c|c|c|c|c|c|}
\hline $\begin{array}{l}\text { At. wts. } P \\
\text { per liter. }\end{array}$ & $\begin{array}{l}\text { Per cent. P as ortho acid. } \\
\text { Per cent. } P \text { as pyro acid. }\end{array}$ & $\begin{array}{r}100 \\
0\end{array}$ & $\begin{array}{l}75 \\
25\end{array}$ & $\begin{array}{l}50 \\
50\end{array}$ & $\begin{array}{l}25 \\
75\end{array}$ & $\begin{array}{r}0 \\
100\end{array}$ \\
\hline \multirow[t]{2}{*}{ O.I } & Found........... & 9650 & 11640 & I 3650 & 15610 & 17690 \\
\hline & Calculated........ & $\ldots$ & I I 660 & 13670 & I 5680 & \\
\hline \multirow[t]{2}{*}{0.05} & Found...... & $6 \mathrm{I} 35$ & 6992 & 7874 & 8726 & 9622 \\
\hline & Calculated........ & $\ldots$ & 7007 & 7878 & $875^{\circ}$ & \\
\hline \multirow[t]{2}{*}{ o.or } & Found. & 2030 & 2154 & 2275 & 2388 & $25 I^{7}$ \\
\hline & Calculated......... & & $2 I^{2}$ & 2274 & 2395 & \\
\hline
\end{tabular}

The degree of the divergence from a linear relation between conductance and composition will be seen by comparing the found values with the calculated ones given in the rows just beneath them. These calculated values were obtained by the expression $x \mathrm{~L}_{0}+(\mathrm{I}-x) \mathrm{L}_{\mathrm{p}}$, where $x$ represents the fraction of the phosphorus present in the mixture as ortho acid, $\mathrm{L}_{0}$ the conductance of the pure ortho acid, and $\mathrm{L}_{p}$ that of the pure pyro acid. It will be seen that the difference is largest in the case of the mixtures with.25 per cent. ortho acid, but that even in this case it averages only about 0.4 per cent.

This opportunity may be also utilized for correcting the following typographical errors in the article on The Ionization Relations of Orthoand Pyrophosphoric Acids and their Sodium Salts, by Abbott and Bray:

On page 742 , line 19 , insert $K_{\mathrm{B}}$ in the denominator of the expression for $K_{3}$.

On page $75 \mathrm{I}$, line $\mathrm{I} 4$, read 353.8 instead of 358.8 .

On page $75 \mathrm{I}$, last two lines, read $K^{\prime}{ }_{1}$, for $K^{\prime}{ }_{2}$ and $K^{\prime}{ }_{2}$ for $K^{\prime}{ }_{1}$.

On page 753 , in Fig. 2, read $\mathrm{Na}_{4} \mathrm{P}_{2} \mathrm{O}_{7}$ in place of $\mathrm{Na}_{4} \mathrm{P}_{4} \mathrm{O}_{3}$.

On page 762 , in Table XXIII, read $\mathrm{H}_{2} \mathrm{PO}_{4}^{-}$in place of $\mathrm{HPO}_{4}^{-}$and $\mathrm{H}_{3} \mathrm{P}_{2} \mathrm{O}_{7}$ - in place of $\mathrm{HP}_{2} \mathrm{O}_{7}^{-}$.

Univ. OF NORTH DAKOTA, Oct., 1910.

[CONTRIBUtion From the Chemical Laboratory OF HaRVard University.]

\title{
A REVISION OF THE ATOMIC WEIGHT OF CALCIUM. I. ANALY- SIS OF CALCIUM BROMIDE.
}

By Theodora W. Richards and OtTo Hönigschmid.

Received October 24, 1910.

Introduction.

During the last fifty years the atomic weight of calcium has been repeatedly investigated, but most of the investigations have not led to

${ }_{1}^{1}$ This Journal, 3r, 729 (I909). 
very favorable results. According to the opinion of Brauner, ${ }^{1}$ one of the most competent of modern critics, only two of the modern researches, namely that by one of the present authors ${ }^{2}$ and that by Hinrichsen, ${ }^{3}$ are to be considered as free from serious error.

The investigation of the atomic weight of calcium was undertaken at Harvard about fifteen years ago, and preliminary determinations were made upon the relation of calcium chloride to silver chloride, resulting in a value for the atomic weight of calcium of 40.085 , if silver and chlorine are given the values of 107.88 and 35.457 , respectively. Hinrichsen, on the other hand, without knowledge of the Harvard work, used the old method of converting calcium carbonate to oxide, obtaining therefrom a much higher value, 40.139. Before Stas' atomic weight of silver (107.93) was found to be in error, the two results seemed to be fairly consistent, but with modern values the difference is considerable.

This difference between the two values suggests the importance of a new determination of this important constant, especially because each of the earlier investigators had used only one method. We accordingly began the investigation with the analysis of calcium bromide, and determined not only the amount of silver needed to precipitate the bromine, but also the amount of silver bromide obtained. As will be seen, the results supported rather the earlier Harvard work than the higher value of Hinrichsen, The outcome of this investigation of the bromide $(\mathrm{Ca}=$ 40.070 ) is slightly lower even than the value obtained from the chloride. Further work upon the chloride has already been begun and it is hoped that further results upon this subject also may be communicated in the near future.

It is a pleasure to express our obligation to the Carnegie Institute of Washington for generous pecuniary assistance, without which much of the apparatus necessary in this investigation could not have been procured.

\section{Preparation of Materials.}

During the earlier work upon the atomic weight of calcium, undertaken fifteen years ago, certain tests were made concerning the adequacy of the purification of calcium nitrate from magnesium and from allied elements.

A concentrated calcium nitrate solution containing some magnesium was treated with milk of lime, and the solution was allowed to stand for three days, being shaken repeatedly. One-tenth liter of the supernatant solution, containing about 25 grams of calcium nitrate, was di[Leipzig].

${ }^{1}$ Brauner, Abegg's "Handbuch der anorganischen Chemie," II, [2] I75 (1905)

${ }^{2}$ Richards, This Journal, 24, 374 (1902); $Z$, anorg. Chem., 31, 27 (1902); "Experimentelle Untersuchungen," 626 (1909).

${ }^{3}$ Hinrichsen, Z. physik. Chem, 39, 31 I (1902); 40, 746 (1902). 
Iuted to 0.3 liter and made strongly alkaline with ammonia. Fifty grams of ammonium chloride were added and the calcium was wholly precipitated hot by excess of ammonium oxalate. No magnesium whatever could be found by the usual phosphate test in the filtrate, showing that the treatment with lime eliminates most of the magnesium; but the test does not conclusively prove that all the impurity is removed, for a trace might have been carried down with the oxalate, and thus have escaped detection. It will presently be shown that the crystallization of the nitrate is an effective means of removing the last traces of magnesium, if any such are present.

Turning now to the removal of barium and strontium from the calcium precipitations, an effort to accomplish this by means of sulphuric acid may first be described. First a 20 per cent. solution of calcium nitrate was treated with a small quantity of sulphuric acid, enough to produce a small precipitate, and this precipitate was tested carefully for strontium and barium with negative results. The successive addition, three times, of small further weights of sulphuric acid likewise gave no test for strontium or barium, in spite of the fact that the solution was known to contain a precipitable amount of strontium and a trace of barium.

There is indeed nothing surprising in this observation; it is owing to the fact that the solubility of any electrolyte in a solvent containing different ions is so much increased that the last traces cannot be eliminated by any ordinary process of precipitation. In order to eliminate the impurity, the desired substance, not the impurity, must be separated out.

Abandoning now these methods of purification, which rest upon a wrong principle, the following test was made of the recrystallization of calcium nitrate. To 200 grams of a fairly pure sample of calcium nitrate were added 2 grams each of barium, strontium and magnesium nitrates. The mixed solution was then concentrated and about three-fourths of the calcium nitrate was crystallized from it by cooling. In the mother liquor strong tests for barium, strontium and magnesium could be obtained, while in the crystals indications of these impurities were much feebler, although still unquestionable. The crystals were dissolved and recrystallized twice. The third mother liquor gave no test whatever for magnesium by means of phosphate solutions in the usual way, or for strontium in the spectroscope, but gave a very faint precipitate with sulphuric acid, showing the presence of barium. The crystals contained much less barium.

It is clear that when the calcium nitrate is recrystallized, these other contaminating nitrates tend strongly toward the mother liquor. Hence it is not surprising that the next crystallization, the fourth, yielded crystals in which no impurity whatsoever could be found by any ordinary 
tests. If four crystallizations can accomplish this, when as much as three per cent. of impurity is present, it is clear that when the initial purity is greater, the product must be exceedingly pure, especially upon still further crystallization; for in the distribution between the crystals and mother liquor, each mother liquor tends to hold the greater part of the contaminating substance. Hence both in the earlier investigation and in the present one, as well as in any other investigation in which pure calcium salts are needed, it was our invariable practice to purify the material through the recrystallization of the nitrate.

Calcium Nitrate,-For the present investigation 2 kilograms of a good quality of marble were dissolved in a small excess of the purest commercial nitric acid and the solution was boiled with chlorine water in order to oxidize the iron-an end effected only very slowly by dilute nitric acid. Pure milk of lime was then added in slight excess in order to precipitate the iron and magnesium. The clear filtrate, after the separation of the impurities in solid form, was evaporated to crystallization; and the nitrate was crystallized five times in porcelain vessels and yet five times more in platinum vessels. Each crop of crystals was thoroughly freed from mother liquor by means of a centrifugal machine, at first of porcelain, afterwards of platinum, so that the final crystals came in contact with nothing but the noble metal.

A second specimen of calcium nitrate was prepared from pure calcium carbonate which had been precipitated from nitrate prepared in the manner above described, but with fewer crystallizations. The carbonate was precipitated by means of ammonium carbonate and was washed by repeated decantations and centrifugal action. It was finally dissolved in the purest nitric acid and the nitrate was crystallized four times from the purest water in platinum vessels and whirled in the platinum centrifuge. This calcium nitrate (Sample 2) was used as the basis for the preparation of most of the calcium bromide.

Calcium Carbonate.-From the solution of calcium nitrate thus prepared the carbonate was precipitated by means of very pure ammonium carbonate. For the preparation of this substance in a state of sufficient purity, the purest ammonium carbonate of commerce was distilled with water by means of a platinum cooler. The distillate was collected in a platinum flask containing thrice distilled water, cooled with ice outside. The precipitation of the calcium carbonate was conducted at $100^{\circ}$ in a platinum vessel and the precipitate was repeatedly washed with the purest water and centrifugally freed from adhering wash water. The pure carbonate was preserved in a platinum dish in a desiccator containing lime made from pure marble, in order to protect it from the access of impurity from glass and from the atmosphere of the laboratory. It 
is needless to say that carbonates are especially liable to be contaminated by traces of any strong acid in their neighborhood.

Hydrobromic Acid.-This acid, which was used in very large amounts, was made by the catalytic action of hot platinum on the mixture of hydrogen and bromine vapor. The bromine was prepared from a mixture of pure bromide and bromate by means of sulphuric acid and was still further purified before it was united with the hydrogen.

The bromide had been made from bromate, and this had been many times recrystallized in order to free it from traces of chloride and chlorate. The bromate, and therefore the bromide, made from it, were almost, if not quite, free from iodine, as far as any ordinary test could indicate. ${ }^{1}$

The purest concentrated sulphuric acid of the laboratory was used for the decomposition of the mixed bromate and bromide, and the reaction was carried out in a flask, into which was ground a glass stopper containing a drop funnel and the tube for leading off the bromine vapor. The flask was partly filled with a cold saturated solution of potassium bromide, and the calculated weight of the bromate was added in solid condition. The exit tube was led into another flask partially filled with the purest water and packed in ice; and the reaction was carried out by the gradual dropping in of the acid and gentle warming. There was no difficulty in regulating the speed of the reaction in this way. In each operation only about 50 grams of bromine were prepared, but as this was repeated twenty times, about a kilogram was finally obtained. The bromine thus prepared was purified by solution in a concentrated solution of calcium bromide (made from the same bromine and pure halogen-free lime, according to Stas' method) and the pure bromine was finally once more distilled.

From this bromine, hydrobromic acid was made, as already stated, through direct union of the components with catalytic acceleration by means of platinum asbestos. The hydrogen was prepared through the action of so-called "hydrone" (an alloy of lead and sodium) upon water. The gas was passed through a drying apparatus filled with stick potash, next through a gas-washing bottle filled with the pure bromine, and finally into a Jena hard-glass tube in which platinum asbestos was heated by means of an electric furnace to about $400^{\circ}$. The hydrobromic acid was led into pure water and cooled by ice. The dilute solution of hydrobromic acid thus made always contained an excess of bromine; upon distillation, this bromine, together with any possible traces of iodine which might have been present, came over first and was discarded. The main

1 These materials were both presented to us by Mr. H. H. Dow, of the Midland Chemical Company, Indiana. We take pleasure in acknowledging our indebtedness to him for his generosity and for the trouble which he took in conducting very carefully the preliminary purification of large amounts of both substances. 
portion of the acid was distilled and condensed by means of a quartz cooler, and the distillate was collected in a quartz flask.

Calcium Bromide.-All the calcium bromide used in the investigation was prepared with hydrobromic acid made in this fashion. The pure specimens of calcium carbonate were in the first place heated in an electric oven until wholly dry and free from traces of ammonium salts which always cling to carbonate prepared as this had been. Even if traces of the ammonium salts remained after this treatment, they would not have been harmful; for they must have been wholly volatilized at the high temperature to which the calcium bromide was subsequently heated. Calcium carbonate thus dried was added in small portions to the pure acid in the quartz fiask as long as evolution of gas continued. The solution was then slightly acidified, transferred to a quartz dish and concentrated upon an electric stove. The solution always became yellow, because traces of calcium nitrate (held by the calcium carbonate) invariably set free corresponding traces of bromine during the evaporation.

In these quartz vessels the bromide was recrystallized; thus from beginning to end neither the factors nor the product of the reaction came into contact with glass. The first specimen of calcium bromide was nine times crystallized, during which process of course the greater part of the salt found its way into the mother liquors, and only substance enough for two analyses (Nos. I6 and I7) was obtained. The substance from the mother liquors, after four recrystallizations, was used for preliminary determinations.

A second preparation of bromide was made out of the second preparation of nitrate in a similar manner except that the bromide was crystallized only four times. This preparation served for all the final analyses with the exception of the two mentioned above.

Before the final crystallization in each case the solution was filtered through a platinum Gooch-Munroe crucible. This is important, for in spite of the greatest possible care traces of dust find their way inevitably into the solution and are held by the crystals, appearing in the fused calcium bromide at the end as minute black specks of carbon. When the salt has been filtered just before the last crystallization, such undesirable irregularities are absent. The efficacy of the process of purification was in every case greatly increased by means of the centrifugal drying of the crystals with the help of a centrifuge composed of swinging platinum funnels driven at a high speed by an electric motor. All the operations of transference and evaporation were effected inside of a glass house erected on the laboratory desk, composed of large glass plates held together by a light framework of wood. These plates had been carefully rounded on the edges with a file and protected by split rubber tube, so as to protect the edges and prevent the rubbing off of small par- 
ticles of glass. An electric stove with a quartz top provided a source of heat for all these operations.

Silver. - The silver was prepared in a manner essentially similar to that used in other recent investigations in Harvard University. It was many times recrystallized as the nitrate, precipitated as pure silver by means of ammonium formate, and fused on boats of pure lime in a stream of pure dry hydrogen. For further particulars previous papers from this laboratory should be consulted. ${ }^{1}$

\section{The Drying and Melting of Calcium Bromide, and the Neutrality of Its Solution.}

The salt crystallizes with six molecules of water of crystallization, and dissolves in this water of crystallization at about $38^{\circ}$. As in the case of other concentrated solutions, of similar kinds, the quantitative expulsion of the water from this solution is a difficult matter. Accordingly, in order to dry the salt, the method was adopted which had been used successfully in the earlier analyses of calcium chloride by one of us. The salt was in the first place dried in a desiccator warmed to a temperature just below the "melting point" of the crystallized salt, pure lime being used as the desiccating agent. The water then escapes with considerable rapidity from the crystals, leaving a quantity of effloresced material whose melting point is above $200^{\circ}$. The evaporation of the water from the crystals is of course much hastened by having the vacuum as perfect as possible; accordingly the vacuum desiccator was thoroughly exhausted at a low temperature by means of a Geryk pump. The salt itself was placed in a flat platinum dish. After remaining about eight days in the desiccator, the temperature in the thermostat containing this apparatus could be raised to 50 or $60^{\circ}$, and after eight days more the dehydration had proceeded so far that the water could be all driven off at a much higher temperature without danger of partial melting and the projection of drops of the solution.

In order to eliminate the last traces of water, the salt was melted as usual in a current of hydrogen mixed with hydrobromic acid. This last substance is necessary to prevent the partial decomposition of the salt by the remaining trace of water at a high temperature. The temperature was raised very slowly so that as much water as possible should be driven off from the salt while it was still in a solid condition, and only at the every end was the temperature raised to the melting point at a red heat. The heating was conducted electrically by means of a ribbon of an alloy of nickel and chromium wound directly around the quartz tube which contained the platinum boat holding the calcium bromide

${ }^{1}$ See especially Richards and Wells, This Journal, 27, 472 (1905). Pub Carnegie Inst., 28, 16 (1905). Z. anorg. Chem., 46, 70 (1905). "Experimentelle Untersuchungen." T. W. Richards [Hamburg, I909], p. 703 . 
to be melted. The progress of the fusion could be watched through the spaces between the ribbon. For twenty minutes the calcium bromide was kept in a fused condition at a medium red heat; and by means of light rapping on the tube the mobile liquid was freed as much as possible from bubbles of gas. When this heating had been finished the hydrogen and hydrobromic acid were shut off, and pure nitrogen was led into the tube, at the same time lowering the temperature to about $400^{\circ}$. After all trace of hydrobromic acid had been driven out, the salt was allowed to cool in a stream of nitrogen, and finally this gas was replaced by pure air. The succession of gases was provided by means of suitably arranged purifiers, all connected by glass stopcocks in a method which has been described in previous communications. The quartz tube was attached to the familiar Harvard "bottling apparatus" by means of which the pure anhydrous bromide was enclosed in a weighing tube in an atmosphere of pure air, and after it had become completely cooled this weighing tube was taken out and placed in a desiccator for at least five hours near the balance by means of which it was to be weighed. Successive weighings of the boat and bottle never differed more than 0.02 milligram. The empty boat and weighing bottle were weighed both before and after the determination. For this purpose they received precisely the same treatment as during an analysis, the boat being ignited in the bottling apparatus, and prepared for weighing just as if it contained calcium bromide. The similarity of treatment affords the best means of avoiding constant errors. That the apparatus served its purpose is clear from the fact that in two cases wherein specimens of melted and weighed bromide were once more melted and weighed, no change exceeding a few hundredths of a milligram was to be observed. The loss of weight of the boat during fusion was never over one-twentieth of a milligram, and sometimes amounted to nothing at all.

The bromide was dissolved in water in a large Erlenmeyer flask capable of holding three liters, with a ground stopper. The details were similar to those in the case of lithium chloride, ${ }^{1}$ except that the testing for neutrality was somewhat different, because calcium bromide is decidedly a less stable salt than most of the other halides which have been studied in this laboratory, and therefore is less easy to obtain in a state of perfect neutrality. When heated with either air or with water, it is certain to lose some bromine and become alkaline. On the other hand, when heated with hydrobromic acid, a trace of this gas is dissolved. This trace is indeed quickly liberated on further fusion in nitrogen, but if the nitrogen is not absolutely pure, there is danger of overstepping the mark and making the salt slightly alkaline in this process. Therefore every

${ }^{1}$ Richards and Willard, ThIs Journal, 32, 4 (1910), Pub. Carnegie Inst. of Washington, $\mathbf{2 5}$. 
sample of calcium bromide must be carefully tested in order to be sure of its perfect neutrality; and because the salt is slightly hydrolyzed in aqueous solution, the only satisfactory method of testing it is to compare the solution of the fused salt with a solution of the same concentration made from the purest calcium bromide many times recrystallized. The latter, if prepared with adequate care, is presumably neutral, for there is no evidence that either hydrobromic acid or lime tend to go into the crystals to any perceptible extent. In order, therefore, to determine the deviation from neutrality of the fused calcium bromide, it was dissolved in a small amount of water, and to it was added ro cc. of a saturated aqueous solution of the slightly soluble methyl red, the only indicator which seemed to serve well for this purpose. To the same volume of a solution containing the crystallized salt, assumed to be neutral, was added a like volume of the solution of methyl red, and the colors of the two solutions were compared. When the colors of the two solutions appeared identical, it was reasonable to infer that the fused salt was perfectly neutral. This occurred in two out of the twelve cases. In most cases the fused salt was found to be either slightly alkaline or slightly acid, but two or three drops of the corresponding hundredth normal solution were enough to restore perfect neutralization, except in one case when five drops were required. The appropriate corrections were of course made in the weighings of fused calcium bromide to eliminate the errors which would otherwise have existed in the results because of this lack of neutrality. The absence of such corrections was one of the chief reasons why the ten preliminary results, not worthy of recording here, varied among themselves by amounts distinctly greater than those to be noted in the final results, recorded later.

After the testing for neutrality, the solution was diluted with a liter of the purest water, and the boat was removed by means of a platinum wire, and very thoroughly washed with all possible care. The analysis of the bromide was then carried out by either one of the two usual methods, namely, either the determination of the exact amount of silver equivalent to the calcium present, or the weighing of the precipitated silver bromide. These two processes were never executed in the same experiment in either of the final series, because each can be done more satisfactorily if attention is concentrated upon it alone.

Balance and Weights.- The weighings were made upon a very good balance of Prussian manufacture which had been thoroughly tested and was easily sensitive to 0. or $\mathrm{mg}$. The gilded brass weights were standardized according to the method usually employed in this laboratory and were often further tested during the progress of the research. As usual, all readings were made by substitution, the substituted tare being an object similar in all respects to that which was being weighed. 
It was necessary to determine anew the specific gravity of calcium bromide, because the published values of this constant are highly discrepant among themselves, difiering to an extent sufficient to influence appreciably the calculation of the true weight of the substance. This determination was carried out in a pycnometer for hygroscopic solids described by Baxter. ${ }^{1}$ Toluene was used as a fluid to be displaced. The sample had been distilled over sodium and was found to have at' $25^{\circ} \mathrm{a}$ density of 0.8605 as the mean of three trials. With this apparatus 2.09625 grams of calcium bromide displaced 0.5378 gram of toluene and 2.974 grams of calcium bromide displaced 0.7629 gram of toluene, the readings being reduced to the vacuum standard. These two determinations give as the specific gravity of calcium bromide the values 3.354 and 3.353 , respectively-results essentially identical.

The vacuum corrections to be applied to silver, silver bromide and calcium bromide, when the readings were conducted in air at normal pressure and at $20^{\circ}$, were taken as $-0.030 \mathrm{mg}$., to.04I $\mathrm{mg}$. and +o. $218 \mathrm{mg}$., respectively, the specific gravity of the brass weights being 8.3. The small changes due to changing pressure and temperature were almost always negligible with these dense substances.

\section{The Determination of the Silver Corresponding to Calcium Bromide.}

A weight of purest silver as nearly as possible equivalent to each portion of carefully weighed bromide (as found in the preliminary experiments) was dissolved in nitric acid in a large flask with bulbs to prevent the escape of spray; and the solution was warmed until the nitrous vapors had been wholly driven out. The solution was then diluted so that it contained not more than Io grams of silver in a liter of water. Every drop of solution was then added in the red light of the dark room to the corresponding bromide solution, little by little, the latter liquid being kept in continual motion. The flask which had contained the silver was of course washed with the greatest care, and the last washings were tested in a nephelometer in order to be sure that every trace of silver had been removed. The mixture had in every case, after precipitation, a volume of about 2.5 liters. It was at first only gently shaken in order to promote complete precipitation without causing the precipitate to cohere in to impenetrable balls; and the mixture in this state was left for twentyfour hours in the dark room covered with a black cloth in order that complete equilibrium might establish itself between the precipitate and the solution. In this way opportunity is given for the washing out of any excess of either reagent which may be occluded by the spongy precipitate. When this is accomplished, the mixture is violently shaken for twenty minutes, the flask being closely stoppered. Under this treat-

'Baxter and Hines, Am. Chem. J, 31, 220 (I904). 
ment the precipitate coheres satisfactorily and deposits itself after short standing so completely that the small particles which cling to the flask above the level of the liquid can easily be washed down into the flask by tilting the latter backwards and forwards, thus causing the clear liquid to wash every portion of the neck.

During this whole time the flask was never opened and especial care was taken that no traces of the precipitate should be held in the control space between the lower end of the stopper and the wall of the flask.

The mixture was now ready for filtration. In order to prevent the ejection of drops of liquid on the removal of the stopper, the air in the flask had been slightly warmed by means of the hands before this stopper had been applied in the first place, so that on opening the flask for filtration air was drawn in and not expelled. Cooling the flask with cold water before opening increased this effect. Upon the removal of the stopper, the neck of the flask and stopper were carefully washed with the purest water; and when the solution had been mixed by two or three rotations of the flask, two samples of the clear mother liquor were taken out and placed in two test tubes. To one was added a solution of a milligram of silver; to the other a corresponding amount of potassium bromide; and both were stirred by suitable bent rods. The comparison of the two tubes in the nephelometer enabled one to see at once whether the bromide or the silver was in excess, and it was easy to be sure of an amount corresponding to 0.05 milligram of silver or of bromide in a liter of the solution. If one or the other was found to be deficient, the corresponding amount was added to the whole mass of the solution, and after new shaking and standing another trial in the nephelometer was made. This process was repeated until the nephelometer showed the end point of the reaction, both tubes exhibiting like opalescence. Incidentally it may be noted once more that the liquids in the nephelometer tubes must be allowed to stand for at least I. 5 to 2 hours after they have been precipitated, otherwise the precipitates do not attain constancy. It is essential that during this time, indeed as promptly as possible after they have been filled, the tubes should be covered with glass caps made of rings sealed to round plates of plane optical glass in order to prevent the access of dust and the resulting serious errors in the outcome. The nephelometer can easily detect I/300o of a milligram of chlorine, and a single mote of dust may contain this amount.

The following table gives the results of the last six analyses, carried out in this way. It is worthy of note that these analyses are all consecutive ones, the thirteenth not having been brought to a conclusion on account of a mistake in weighing the silver. No finished analysis was rejected: 


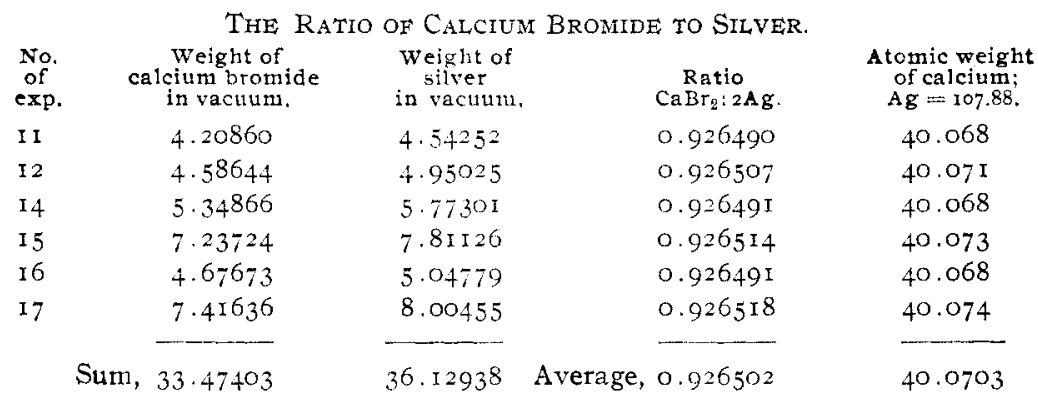

From these six analyses it would appear that the atomic weight of calcium is to be taken as 40.070 , if silver is taken as 107.88 (the present international value). The extremes 40.068 and 40.074 deviate but slightly from the mean, and the so-called "probable error" of the average is only 0.0007 . The series is as concordant as could reasonably be expected, and except for one possibly insignificant fact would go entirely unchallenged. This fact is the hitherto unexplained circumstance that the experiments where larger quantities of material were used gave slightly higher results than those where smaller quantities were used. The same circumstance is to be noted in the second series of results to be recorded shortly. The agreement of the verdict of these two series may be merely a coincidence, and in any case the deviation is so slight as to cause uncertainty only in the third decimal place of the atomic weight. If, as is probable, the results with the larger quantities are somewhat more accurate, the value 40.07 may be slightly too low; but the true value is not likely to be as high as 40.08 .

A more reassuring conclusion may be drawn from another aspect of the results. It is satisfactory that the two different preparations of bromide gave results essentially identical. The specimen recrystallized nine times, which was used in analyses $I 6$ and $I 7$, gave as the mean value $40.07 \mathrm{I}$, whereas that which had been recrystallized four times (used in analyses II to I $_{5}$ ) gave 40.070 , a result essentially identical. This seems to indicate that both preparations were really pure; for it is not probable that if impurities had been present a product crystallized nine times would have shown precisely the same result as one crystallized only four times, especially when one considers that the recrystallization was carried out with the help of an energetic centrifuge.

\section{The Determination of the Relation of Calcium Bromide to Silver Bromide.}

The method of precipitation of silver bromide for weighing was essentially similar to the method used in previous cases in this laboratory. Only a very slight excess of silver was used in precipitating in order to avoid occlusion of silver nitrate. The precipitation was accomplished 
in the manner just described and a platinum Gooch-Munroe crucible was used for filtration. ${ }^{1}$ The precipitate was first washed seven to ten times by decantation with small quantities of the purest water, and finally was washed upon the filtering crucible by means of a jet driven by hydrostatic pressure. Both mother liquor and wash water were preserved separately and carefully tested in the nephelometer for the traces of bromide which they might contain. The mother liquor rarely contained a trace of bromide, the wash water usually about 0.2 milligram per liter. The filtration was conducted under a glass roof, which served to protect the crucible from dust. The flask in which the precipitation had been conducted was finally washed with a small quantity of the purest freshly distilled, concentrated ammonia, in order to dissolve any trace of silver bromide which might have escaped transference into the crucible. After neutralization with the purest nitric acid this mixture too was tested in the nephelometer with excess of silver solution. In making the nephelometric tests, comparison was made in each case with a standard prepared in precisely the same way.

As usual, the dry precipitate, after having been weighed, was transferred to a small porcelain crucible, weighed again, cautiously heated, and weighed for the third time. The fused mass, which was distributed over the inside surface of the crucible by gently rotating the crucible while inclined at a small angle, was a clear yellow upon cooling and gave evidence of being very pure. The loss during melting was never more than 0.004 per cent. and was usually much less.

These rather complicated processes are subject to somewhat greater possibilities of error than the much simpler comparison with silver, but the agreement of the results was, as a matter of fact, about the same as before. The six analyses recorded in this table represent a series from which none is omitted. They were carried out after the necessary experience gained in preliminary determinations.

The Ratio of Calcium Bromide to Silver Bromide.

\begin{tabular}{|c|c|c|c|c|}
\hline $\begin{array}{l}\text { No. } \\
\text { of } \\
\text { exp. }\end{array}$ & $\begin{array}{l}\text { Weight of } \\
\text { calcium bromide } \\
\text { in vacuum. }\end{array}$ & $\begin{array}{l}\text { Weight of } \\
\text { silver bromide } \\
\text { in vacuum. }\end{array}$ & $\begin{array}{c}\text { Ratio } \\
\mathrm{CaBF_{2 }}: 2 \mathrm{AgBr}\end{array}$ & $\begin{array}{c}\text { A tomic weight } \\
\text { of calcium } \\
\text { A g Br }=187.795 .\end{array}$ \\
\hline I 8 & I0.I 859 I & I9. 13778 & $0.53224 \mathrm{I}$ & 40.073 \\
\hline 19 & $7 \cdot 92400$ & $14.888 \mathrm{IO}$ & 0.532237 & 40.072 \\
\hline 20 & 6.78048 & I $2.7396 \mathrm{I}$ & 0.532236 & 40.072 \\
\hline $2 \mathrm{I}$ & 6.45970 & 12.13702 & $0.53223 \mathrm{I}$ & 40.070 \\
\hline 22 & 5.95390 & I I . I 8684 & 0.532225 & 40.067 \\
\hline 23 & $5 \cdot 15998$ & 9.69513 & 0.532225 & 40.067 \\
\hline & $1,4^{2} .46397$ & $79 \cdot 7844^{8}$ & 0.5322323 & 40.0702 \\
\hline
\end{tabular}

A glance at this table shows that the figures recorded in it afford a

${ }^{1}$ Richards and Mueller, Z. anorg. Chem., 53, 438 (1907); This Journal, 29, 65I (1907). 
complete confirmation of the results contained in the first table, although these were reached by a different road. Indeed the final averages $(40.0702$ and 40.0703 ) are almost precisely the same in the two cases, far within the almost equal "probable errors" $(0.0007)$ of the two series. In the present case, as in the other, the greatest deviation from the mean is about 3 in the third decimal place of the atomic weight of calcium, or about $I$ in 70,000 in the total weight of the calcium bromide; this seems to be about the limit of reasonable accuracy in such experimentation. The result, as in the other case, is probably to be regarded as a minimum value, and it seems fairly safe to conclude that the atomic weight of calcium is between 40.07 and 40.08 .

Obviously, because the two series give identical results, the atomic weight of bromine computed from the two average results is equal to that assumed in the calculation of each series, namely, 79.916 , if silver is 107.88 . This shows that the calcium bromide was free from chloride, and that the occlusion of other salts by the silver bromide must have been imperceptible.

We have already obtained some interesting confirmatory results with calcium chloride, which will soon be ready for publication, and hope to continue the study of this atomic weight and to settle such points as remain obscure.

\section{Summary.}

The results of this paper may be stated in a few words, as follows:

Methods for the preparation, dehydration, and melting of pure calcium bromide were devised.

Calcium bromide proved to be less stable on melting than the corresponding barium and strontium salts. By comparison with pure crystallized salt with the help of methyl red, the very slight deviations of the several specimens of melted bromide from exact neutrality were determined in their aqueous solutions.

The density of melted calcium bromide was found to be 3.353 at $25^{\circ}$

33.47403 grams (in vacuum) of calcium bromide required 36.12938 grams (in vacuum) of silver for complete precipitation. Again, 42.46397 grams (in vacuum) of calcium bromide yielded 79.78448 grams (in vacuum) of silver bromide, each set of figures being the sum of the quantities used in six consecutive determinations. The two ratios give essentially the same value for the atomic weight of calcium, 40.070 , if silver is taken as 107.88 . The value becomes 40.066 if silver is taken as 107.87. This result is probably a minimum value; but it is improbable that the true one exceeds 40.08 .

The investigation shows that the preliminary Harvard work yielded a value for the atomic weight of calcium much more probable than that found by the only other modern investigator. 